\title{
Finite-Time Stability of Linear Time-Varying Systems with Jumps: Analysis and Controller Design
}

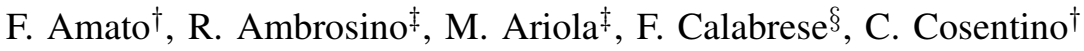

\begin{abstract}
This paper deals with the finite-time stability problem for continuous-time linear time-varying systems with finite jumps. This class of systems arises in many practical applications and includes, as particular cases, impulsive systems and sampled-data control systems. The paper provides a necessary and sufficient condition for finite-time stability, requiring a test on the state transition matrix of the system under consideration, and a sufficient condition involving two coupled differential/difference linear matrix inequalities. The sufficient condition turns out to be more efficient from the computational point of view. Moreover, it is the starting point for solving the stabilization problem, namely for finding a state feedback controller which finite-time stabilizes the closed loop system. Some examples illustrate the effectiveness of the proposed approach.
\end{abstract}

\section{INTRODUCTION}

The concept of finite-time stability (FTS) dates back to the Sixties, when it was introduced in the control literature [11], [8]. A system is said to be finite-time stable if, given a bound on the initial condition, its state does not exceed a certain threshold during a specified time interval. It is important to recall that FTS and Lyapunov Asymptotic Stability (LAS) are independent concepts; indeed a system can be FTS but not LAS, and vice versa. While LAS deals with the behavior of a system within a sufficiently long (in principle infinite) time interval, FTS is a more practical concept, useful to study the behavior of the system within a finite (possibly short) interval, and therefore it finds application whenever it is desired that the state variables do not exceed a given threshold (for example to avoid saturations or the excitation of nonlinear dynamics) during the transients.

In [4] and [3] a sufficient condition for FTS and finitetime stabilization of continuous-time linear time-invariant systems is provided, by using an approach based on Lyapunov functions theory; such condition requires the solution of a feasibility problem involving Linear Matrix Inequalities (LMIs). A different approach, which is reminiscent of optimal control techniques and is also applicable to linear time-varying systems, has been proposed in [2] and [1]. In the time-invariant case, the main result of [1] turns out to be less conservative than the condition provided in [4], but

$\dagger$ F. Amato and C. Cosentino are with the School of Computer Science and Biomedical Engineering, Università degli Studi Magna Græcia di Catanzaro, Via T. Campanella 115, 88100 Catanzaro, Italy.

$\ddagger$ R. Ambrosino and M. Ariola are with the Dipartimento per le Tecnologie, Università degli Studi di Napoli Parthenope, Via Medina 40, 80133 Napoli, Italy.

$\S$ F. Calabrese is with the Dipartimento di Informatica e Sistemistica, Università degli Studi di Napoli Federico II. it is computationally more demanding since it involves the solution of a Differential Linear Matrix Inequality (DLMI).

In this paper we consider the class of linear timevarying systems with finite state jumps. The concept of linear systems with jumps was firstly proposed by Sun, Nagpal and Khargonekar in [10]. Roughly speaking, such system is a linear continuous-time system whose state undergoes finite jump discontinuities at discrete instants of time. Obviously, linear systems with jumps contain the class of continuous-time linear systems, but capture many other cases of practical interest in engineering applications, e.g. impulsive control systems [12], hybrid control systems [5] and sampled data control systems. In particular, systems with jumps were introduced as a suitable framework for representing closed loop sampled-data systems in which the inter-sample behavior is of interest.

This work follows the spirit of [2] and [1] to derive the main results for FTS analysis and design of linear systems with jumps. The first contribution of the paper is a necessary and sufficient condition for FTS. It requires the computation of the state transition matrix of the given system, a numerically hard problem except for timeinvariant systems; moreover this result is not useful for design purposes. Therefore we also provide a sufficient condition for FTS, which requires the solution of two coupled differential/difference Lyapunov inequalities; the Lyapunov inequalities can be turned into differential/difference linear matrix inequalities (D/DLMIs) which can be efficiently solved with many existing software packages. Moreover the sufficient condition is the starting point to solve the finite-time stabilization problem. To this regard, a sufficient condition, again based on D/DLMIs, for the existence of a finite-time stabilizing state feedback controller is provided. Some examples illustrate the application of the proposed technique.

The paper is organized as follows: in Section II the definition of FTS for a linear system with jumps is precisely stated, some preliminary results are provided and the main problems we will deal with in the paper are defined. In Section III the analysis conditions are given; they are thoroughly discussed and illustrated through a numerical example. In Section IV the conditions for the existence of a stabilizing state feedback controller are provided. Finally some concluding remarks are given in section $\mathrm{V}$. 


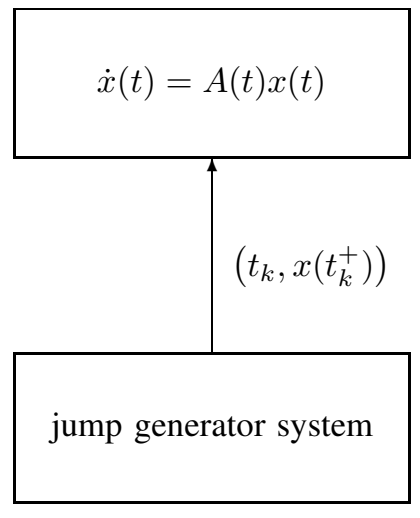

Fig. 1. Linear system with jumps.

\section{Problem Statement}

Let us consider the continuous-time linear time-varying system described by

$$
\dot{x}(t)=A(t) x(t), \quad t \geq 0, \quad x(0)=x_{0},
$$

where $A(\cdot): t \in[0,+\infty) \mapsto \mathbb{R}^{n \times n}$ is continuous.

We assume that the evolution of the state vector $x(t)$ may be right discontinuous at the time instants $t_{k}>0$, $k=1,2, \ldots, v$, where the state may exhibit a finite jump from $x\left(t_{k}\right)$ to $x\left(t_{k}^{+}\right) \neq x\left(t_{k}\right)$, according to the decision of a "jump generator system" (see Figure 1 ). Since $A(\cdot)$ is continuous, $x(\cdot)$ cannot have a finite escape time. Therefore $x(\cdot)$ turns out to be a piecewise continuous function, i.e. in each compact interval of $[0,+\infty)$ it has a finite number of discontinuities and the right and left limits at the discontinuity points are both finite.

The configuration shown in Figure 1 captures many cases of practical interest. For example if $x\left(t_{k}^{+}\right)$is generated through an asynchronous impulsive input entering the system, we reobtain the class of impulsive control systems [12], while if $x\left(t_{k}^{+}\right)$is computed according to a given algorithm (for example it is the output of a discrete event system [6]) the system depicted in Figure 1 falls in the category of hybrid control systems [5].

According to [10], we consider the case when the jump of the state variables is computed as the output of a discretetime system described by the following difference equation

$$
x\left(t_{k}^{+}\right)=A_{d}(k) x\left(t_{k}\right), \quad k=1,2, \ldots, v,
$$

where $A_{d}(\cdot): k \mapsto \mathbb{R}^{n \times n}$. Note that, according to [10], sampled-data systems can be described through (1) and (2) (see also Section III-A).

In this paper we deal with the behavior of the system (1)(2) within a finite interval $] 0, T]$ and let $r$ such that $T \in$ $\left[t_{r}, t_{r+1}\right]$. The solution of system (1)-(2) in the considered interval is given by

$$
x(t)=\Phi(t, 0) x_{0}, \quad t \in[0, T],
$$

where the matrix function $\Phi(t, \tau)$ is the state transition matrix of system (1)-(2). The transition matrix turns out to be piecewise continuous with possible right discontinuities at the time instants $t_{k}, k=1,2, \ldots, r$. In the first interval, $\Phi(t, \tau)$ satisfies the following matrix differential equation

$$
\begin{aligned}
\frac{\partial}{\partial t} \Phi(t, 0) & =A \Phi(t, 0), \quad t \in\left[0, t_{1}\right] \\
\Phi(0,0) & =I
\end{aligned}
$$

in the following intervals we have

$$
\begin{aligned}
\frac{\partial}{\partial t} \Phi\left(t, t_{k}^{+}\right) & \left.\left.=A \Phi\left(t, t_{k}^{+}\right), \quad t \in\right] t_{k}, t_{k+1}\right], \\
k & =1,2, \ldots, r-1 \\
\Phi\left(t_{k}^{+}, t_{k}^{+}\right) & =A_{d}(k) \Phi\left(t_{k}, t_{k-1}^{+}\right), \quad k=1,2, \ldots, r-1,
\end{aligned}
$$

where $t_{0}^{+}=t_{0}:=0$ (obviously at $t_{0}=0$ there is no discontinuity). Finally in the last interval we have

$$
\begin{aligned}
\frac{\partial}{\partial t} \Phi\left(t, t_{r}^{+}\right) & \left.\left.=A \Phi\left(t, t_{r}^{+}\right), \quad t \in\right] t_{r}, T\right] \\
\Phi\left(t_{r}^{+}, t_{r}^{+}\right) & =A_{d}(r) \Phi\left(t_{r}, t_{r-1}^{+}\right) .
\end{aligned}
$$

In the following we extend the definition of FTS [11], [4], to the class of linear systems with jumps in the form (1)-(2).

Definition 1 (FTS of linear systems with jumps): Given a positive number $T$, a positive definite matrix $R$, a positive definite matrix-valued function $\Gamma(\cdot)$ defined over $[0, T]$, with $\Gamma(0)<R$, system (1)-(2) is said to be finite-time stable with respect to $(T, R, \Gamma(\cdot))$, if

$$
x_{0}^{T} R x_{0} \leq 1 \Rightarrow x(t)^{T} \Gamma(t) x(t)<1 \quad \forall t \in[0, T] .
$$

Remark 1: The definition can be interpreted in terms of ellipsoidal domains. The set defined by $x_{0}^{T} R x_{0} \leq 1$ contains all the admissible initial states. The inequality $x(t)^{T} \Gamma(t) x(t) \leq 1$, instead, defines a time-varying ellipsoid that bounds the state trajectory over the interval $[0, T]$.

\section{MAIN RESUlts: AnALYSIS}

The following theorem provides a necessary and sufficient condition for FTS of system (1)-(2) involving the transition matrix.

Theorem 1: System (1)-(2) is FTS with respect to $(T, R, \Gamma(\cdot))$ iff for all $t \in[0, T]$

$$
\Phi(t, 0)^{T} \Gamma(t) \Phi(t, 0)<R .
$$

Proof: Assume that (5) holds and let $x_{0}^{T} R x_{0} \leq 1$. Then

$$
\begin{aligned}
x(t)^{T} \Gamma(t) x(t) & =x_{0}^{T} \Phi(t, 0)^{T} \Gamma(t) \Phi(t, 0) x_{0} \\
& <x_{0}^{T} R x_{0} \leq 1 .
\end{aligned}
$$

Therefore system (1)-(2) is FTS.

Conversely, assume by contradiction that system (1)-(2) is FTS and that for some $\bar{t}, \bar{x}$

$$
\bar{x}^{T} \Phi(\bar{t}, 0)^{T} \Gamma(\bar{t}) \Phi(\bar{t}, 0) \bar{x} \geq \bar{x}^{T} R \bar{x} .
$$

Now let 
where $\lambda$ is such that

$$
x(0)^{T} R x(0)=1 .
$$

Then (6) implies that

$$
x(0)^{T} \Phi(\bar{t}, 0)^{T} \Gamma(\bar{t}) \Phi(\bar{t}, 0) x(0) \geq 1 .
$$

Therefore

$$
x(\bar{t})^{T} \Gamma(\bar{t}) x(\bar{t})=x(0)^{T} \Phi(\bar{t}, 0)^{T} \Gamma(\bar{t}) \Phi(\bar{t}, 0) x(0) \geq 1,
$$

which contradicts the initial assumption that system (1)-(2) be FTS.

The condition in Theorem 1 may be difficult to apply, unless we are in the time-invariant case, because it requires the computation of the transition matrix. Moreover (5) is not useful for design purposes.

For these reasons, we provide an alternative condition for FTS which involves two coupled differential/difference Lyapunov inequalities. The condition stated in the theorem, however, is only sufficient and therefore it introduces a certain degree of conservativeness in the FTS analysis.

Theorem 2: Assume that the coupled differential/difference Lyapunov inequalities

$$
\begin{aligned}
& \dot{P}(t)+A(t)^{T} P(t)+P(t) A(t)<0, \\
& \quad t \in] 0, T], \quad t \neq t_{k}, \quad k=1,2, \ldots, r \\
& P\left(t_{k}\right)>A_{d}(k)^{T} P\left(t_{k}^{+}\right) A_{d}(k), \quad k=1,2, \ldots, r \\
& P(t) \geq \Gamma(t), \quad \forall t \in[0, T], \\
& P(0)<R,
\end{aligned}
$$

admits a piecewise continuously differentiable symmetric solution $P(\cdot)$; then system (1)-(2) is FTS with respect to $(T, R, \Gamma(\cdot))$.

Proof: Let $t \in[0, T], \tau \in[0, t]$ and $V(\tau, x)=$ $x^{T} P(\tau) x$. Then, if $\tau \neq t_{k}, k=1, \ldots, r_{t}$, the derivative of $V$ along the trajectories of system (1) yields

$$
\dot{V}(\tau, x)=x^{T}\left(\dot{P}(\tau)+A(\tau)^{T} P(\tau)+P(\tau) A(\tau)\right) x,
$$

which is negative definite by virtue of (7a).

At the discontinuity points we have

$V\left(t_{k}^{+}, x\right)-V\left(t_{k}, x\right)=x^{T}\left(A_{d}(k) P\left(t_{k}^{+}\right) A_{d}(k)-P\left(t_{k}\right)\right) x$,

which is negative definite in view of (7b).

We can conclude that $V(\tau, x)$ is strictly decreasing along the trajectories of system (1)-(2) in the interval $[0, T]$; hence, given $x_{0}$ such that $x_{0}^{T} R x_{0} \leq 1$, we have

$$
\begin{aligned}
x(t)^{T} \Gamma(t) x(t) & \leq x(t)^{T} P(t) x(t) \quad \text { by }(7 \mathrm{c}) \\
& <x(0)^{T} P(0) x(0) \\
& <x(0)^{T} R x(0) \leq 1 \quad \text { by }(7 \mathrm{~d}) ;
\end{aligned}
$$

the proof follows from the arbitrariness of $t$.

Remark 2: Theorem 2 reduces the FTS analysis to a feasibility problem in the matrix variable $P(\cdot)$ involving two coupled differential/difference linear matrix inequalities (D/DLMIs), namely (7a) and (7b), a LMI to be tested for

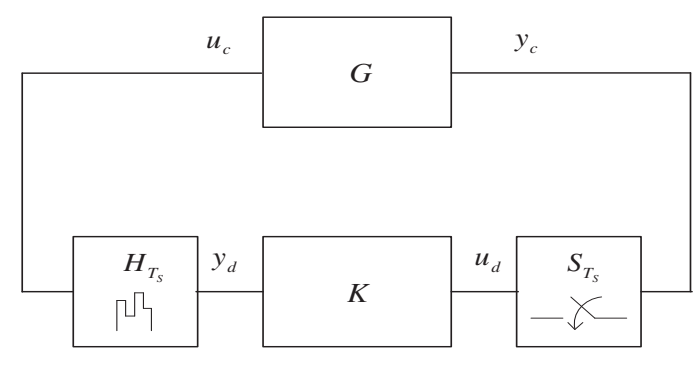

Fig. 2. Sampled-data-system

all $t \in[0, T]$ (inequality (7c)) and the LMI (7d). When the structure of the matrix $P(\cdot)$ is fixed, for example piecewise affine (see Example 1 below), the feasibility problem can be turned into a classical optimization problem involving LMIs [7].

The example in the next section illustrates the application of Theorem 2; since the system we deal with in this case is time-invariant, the example is also useful to perform a comparison between the necessary and sufficient condition stated in Theorem 1 and the sufficient condition provided in Theorem 2.

\section{A. Sampled-data systems as linear systems with jumps}

In this subsection we show how a sampled-data system can be expressed as a linear system with finite jumps.

Consider the sampled-data feedback system in Figure 2 where $G$ is a linear continuous-time, time-invariant plant described by

$$
\begin{aligned}
\dot{x_{c}} & =A_{c} x_{c}+B_{c} u_{c} \\
y_{c} & =C_{c} x_{c},
\end{aligned}
$$

where $x_{c} \in \mathbb{R}^{n}, u_{c} \in \mathbb{R}^{m}, y_{c} \in \mathbb{R}^{o}, x_{c}(0)=x_{c 0}$, and $K$ is a linear discrete-time, time-invariant controller

$$
\begin{aligned}
x_{d}(k+1) & =A_{d} x_{d}(k)+B_{d} u_{d}(k) \\
y_{d}(k) & =C_{d} x_{d}(k)+D_{d} u_{d}(k),
\end{aligned}
$$

where $x_{d} \in \mathbb{R}^{p}, u_{d} \in \mathbb{R}^{o}, y_{d} \in \mathbb{R}^{m}, x_{d}(0)=0$.

The block labeled $S_{T_{s}}$ represents the sampling operator with period $T_{s}$ such that

$$
S_{T_{s}}: y_{c} \rightarrow\left(S_{T_{s}} y_{c}\right):\left(S_{T_{s}} y_{c}\right)(k)=y_{c}\left(k T_{s}\right),
$$

and the block denoted by $H_{T_{s}}$ represents the zero order hold operator with time period $T_{s}$ defined as

$$
\begin{array}{r}
H_{T_{s}}: y_{d} \rightarrow\left(H_{T_{s}} y_{d}\right):\left(H_{T_{s}} y_{d}\right)(t)=y_{d}(k), \\
k T_{s}<t \leq(k+1) T_{s} .
\end{array}
$$

Observe that

$$
\begin{aligned}
u_{d}(k) & =y_{c}\left(k T_{s}\right)=C_{c} x_{c}\left(k T_{s}\right) \\
u_{c}(t) & =y_{d}(k), \quad k T_{s}<t \leq(k+1) T_{s} .
\end{aligned}
$$


Such an interconnection can be reduced to the following state space representation

$$
\begin{aligned}
\dot{x}(t) & =A(t) x(t), \quad x(0)=x_{0} \\
t \geq 0, \quad t & \neq k T_{s}, \quad k=1,2, \ldots \\
x\left(k T_{s}^{+}\right) & =A_{d}(k) x\left(k T_{s}\right), \quad k=1,2, \ldots
\end{aligned}
$$

by letting

$$
\begin{aligned}
x_{1}(t) & =x_{c}(t) \quad \forall t \\
x_{2}(t) & =y_{d}(k) \quad k T_{s}<t \leq(k+1) T_{s} \\
x_{3}(t) & =x_{d}(k+1) \quad k T_{s}<t \leq(k+1) T_{s} \\
x(0) & =\left(\begin{array}{lll}
x_{c 0} & 0 & 0
\end{array}\right)^{T} \\
A & =\left(\begin{array}{ccc}
A_{c} & B_{c} & 0 \\
0 & 0 & 0 \\
0 & 0 & 0
\end{array}\right) \quad A_{d}=\left(\begin{array}{ccc}
I & 0 & 0 \\
D_{d} C_{c} & 0 & C_{d} \\
B_{d} C_{c} & 0 & A_{d}
\end{array}\right) .
\end{aligned}
$$

Thus, the sampled-data system can be expressed as a linear system with finite jumps where $t_{k}=k T_{s}$.

Remark 3: If we consider a sampled-data static state feedback system, i. e. $y_{d}(k)=D_{d} u_{d}(k)$ with $u_{d}(k)=$ $x_{c}\left(k T_{s}\right)$, the state variables of the corresponding linear system with jumps (15a) reduce to

$$
\begin{array}{ll}
x_{1}(t)=x_{c}(t) & \forall t \\
x_{2}(t)=y_{d}(k) & k T_{s}<t \leq(k+1) T_{s},
\end{array}
$$

and

$$
A=\left(\begin{array}{cc}
A_{c} & B_{c} \\
0 & 0
\end{array}\right) \quad A_{d}=\left(\begin{array}{cc}
I & 0 \\
D_{d} & 0
\end{array}\right)
$$

Example 1: Consider the sampled-data static state feedback system, made up of the continuous-time linear plant $G$, defined by the matrices

$$
A_{c}=\left(\begin{array}{cc}
0 & 1 \\
-15 & -0.2
\end{array}\right) \quad B_{c}=\left(\begin{array}{l}
0 \\
1
\end{array}\right) \quad C_{c}=\operatorname{diag}(1,1)
$$

and the state feedback controller

$$
K=\left(\begin{array}{ll}
0.0333 & 0.8519
\end{array}\right),
$$

designed through LQ optimization, sampling and hold blocks having $T_{s}=0.025 \mathrm{~s}$. Theorem 2 is exploited to analyze the FTS of the closed-loop linear system with jumps with respect to $(T, R, \Gamma)$, with $T=1 \mathrm{~s}$,

$$
\Gamma=\left(\begin{array}{ccc}
0.1 & 0 & 0 \\
0 & 0.003 & 0 \\
0 & 0 & 0.02
\end{array}\right), \quad R=\left(\begin{array}{ccc}
0.3 & 0 & 0 \\
0 & 0.02 & 0 \\
0 & 0 & 50
\end{array}\right) \text {. }
$$

In order to recast in terms of LMIs the conditions provided in Theorem 2, the matrix function $P(\cdot)$ has been assumed piecewise affine, that is

$$
\begin{aligned}
P(0) & =\Pi_{1}^{0} \\
P(t) & =\Pi_{k}^{0}+\Pi_{k}^{s}\left(t-(k-1) T_{s}\right), \\
& \left.\quad k \in \mathbb{N}: k \leq \bar{k}, \quad t \in](k-1) T_{s}, k T_{s}\right] \\
P(t) & \left.\left.=\Pi_{k}^{0}+\Pi_{k}^{s}\left(t-\bar{k} T_{s}\right), \quad t \in\right] \bar{k} T_{s}, T\right]
\end{aligned}
$$

where $\bar{k}=\max \left\{k \in N: k<T / T_{s}\right\}$.

Exploiting the Matlab LMI toolbox [9], it is possible to find matrices $\Pi_{k}^{s}$ and $\Pi_{k}^{0}, k=1,2, \ldots, r$ such that $P(\cdot)$ verifies the conditions of Theorem 2 . Therefore we can conclude that the sampled data system with matrices (16)(17) is FTS with respect to $(T, R, \Gamma)$.

Example 2: In order to measure the conservativeness of the sufficient condition stated in Theorem 2 with respect to the necessary and sufficient condition stated in Theorem 1, let us consider the same sampled-data feedback system given in the previous example. We computed the maximum $\epsilon$ such that the system is FTS with respect to $(T, R / \epsilon, \Gamma)$. The exact value of $\epsilon$, computed by using Theorem 1 , is $\epsilon_{\text {true }}=1.74$ while its estimated value, obtained through Theorem 2, is $\epsilon_{\text {suff }}=1.68$. Therefore, in this case the degree of conservativeness is about $3 \%$.

\section{State Feedback Design}

In this section the analysis result will be exploited to derive operative conditions for the design of a state feedback controller guaranteeing the FTS of a given linear system with jumps.

Problem 1 (Finite-time Control via State Feedback): Consider the linear time-varying system

$$
\begin{array}{r}
\dot{x}(t)=A(t) x(t)+B(t) u(t), \quad x(0)=x_{0} \\
t \geq 0, t \neq t_{k}, k=1,2, \ldots, r \\
x\left(t_{k}^{+}\right)=A_{d}(k) x\left(t_{k}\right), \quad k=1,2, \ldots, r,
\end{array}
$$

where $u(t)$ is the control input. Given a positive number $T$, a positive definite matrix $R$, a positive definite matrixvalued function $\Gamma(\cdot)$ defined over $[0, T]$, with $\Gamma(0)<R$, find a state feedback controller in the form

$$
u(t)=K(t) x(t)
$$

such that the closed loop system obtained by the interconnection of (19) and (20), namely

$$
\begin{gathered}
\dot{x}(t)=(A(t)+B(t) K(t)) x(t), x(0)=x_{0} \\
t \geq 0, t \neq t_{k}, k=1,2, \ldots, r \\
x\left(t_{k}^{+}\right)=A_{d}(k) x\left(t_{k}\right), \quad k=1,2, \ldots, r,
\end{gathered}
$$

is FTS with respect to $(T, R, \Gamma(\cdot))$.

Theorem 3: Problem 1 is solvable if there exist a piecewise continuously differentiable symmetric matrix-valued function $Q(\cdot)$ and a matrix-valued function $L(\cdot)$ such that

$$
\begin{aligned}
& -\dot{Q}(t)+A(t) Q(t)+Q(t) A(t)^{T}+L(t)^{T} B(t)^{T}+ \\
& \quad+B(t) L(t)<0, \quad t \in] 0, T], t \neq t_{k}, k=1,2, \ldots, r
\end{aligned}
$$

$$
\left(\begin{array}{cc}
-Q\left(t_{k}^{+}\right) & A_{d}(k) Q\left(t_{k}\right) \\
Q\left(t_{k}\right) A_{d}(k)^{T} & -Q\left(t_{k}\right)
\end{array}\right)<0, \quad k=1,2, \ldots, r
$$

$Q(t) \leq \Gamma^{-1}(t) \quad \forall t \in[0, T]$

$Q(0)>R^{-1}$. 
In this case a controller gain which solves Problem 1 is $K(t)=L(t) Q^{-1}(t)$.

Proof: From Theorem 2 it follows that Problem 1 admits a solution if there exist a piecewise continuously differentiable symmetric matrix function $P(\cdot)$ and a matrix function $K(\cdot)$ such that

$$
\begin{aligned}
& \dot{P}(t)+(A(t)+B(t) K(t))^{T} P(t) \\
& +P(t)(A(t)+B(t) K(t))<0 \\
& t \in] 0, T], t \neq t_{k}, k=1,2, \ldots, r \\
& P\left(t_{k}\right)>A_{d}(k)^{T} P\left(t_{k}^{+}\right) A_{d}(k), \quad k=1,2, \ldots, r \\
& P(t) \geq \Gamma(t), \quad \forall t \in[0, T], \\
& P(0)<R \text {. }
\end{aligned}
$$

Now pre and post-multiply (23a) by $P^{-1}(t)=: Q(t)$. Condition (22a) is obtained noticing that

$$
\dot{Q}(t)=-Q(t) \dot{P}(t) Q(t)
$$

and letting $L(t)=K(t) Q(t)$. Conditions (22c) and (22d) are easily derived from (23c) and (23d) respectively. Finally, condition (23b) can be rewritten as (22b) by using Shur Complements.

Example 3: Consider the second order linear system with jumps (19) defined by the matrices

$$
\begin{aligned}
A & =\left(\begin{array}{cc}
0 & 1 \\
-1 & -1
\end{array}\right) \quad B=\left(\begin{array}{l}
0 \\
1
\end{array}\right) \\
A_{d} & =\left(\begin{array}{cc}
1.1 & 0 \\
0 & 1.1
\end{array}\right)
\end{aligned}
$$

with $t_{k}=k T_{s}, T_{s}=0.1 \mathrm{~s}$. Theorem 3 is exploited to design a state feedback controller which guarantees the FTS of the closed-loop linear system with jumps with respect to $(T, R, \Gamma)$, with

$$
\begin{aligned}
T & =1 s, \\
\Gamma & =\left(\begin{array}{ll}
1 & 0 \\
0 & 1
\end{array}\right), \\
R & =\left(\begin{array}{cc}
2.5 & 0 \\
0 & 2.5
\end{array}\right) .
\end{aligned}
$$

We also added a constrain on the control input

$$
\|u(t)\| \leq 10, \quad t \in[0, T]
$$

and implemented this constrain as LMI (see [7] p. 103)

$$
\left(\begin{array}{cc}
Q(t) & L(t)^{T} \\
L(t) & 100 I
\end{array}\right) \geq 0, \quad t \in[0, T]
$$

In order to recast in terms of LMIs the conditions provided in Theorem 3 , the matrix-valued functions $P(\cdot)$

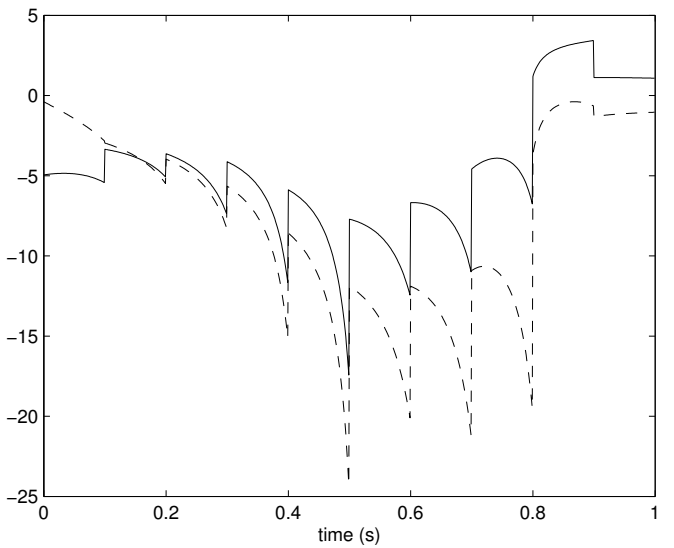

Fig. 3. Scalar functions $K_{1}(t)$ (solid line) and $K_{2}(t)$ (dashed line).

and $L(\cdot)$ have been assumed piecewise affine, that is

$$
\begin{aligned}
P(0)= & \Pi_{1}^{0} \\
P(t)= & \Pi_{k}^{0}+\Pi_{k}^{s}\left(t-(k-1) T_{s}\right), \\
& \left.\quad k \in \mathbb{N}: k \leq \bar{k}, \quad t \in](k-1) T_{s}, k T_{s}\right] \\
P(t)= & \left.\left.\Pi_{k}^{0}+\Pi_{k}^{s}\left(t-\bar{k} T_{s}\right), \quad t \in\right] \bar{k} T_{s}, T\right] \\
L(0)= & \Lambda_{1}^{0} \\
L(t)= & \Lambda_{k}^{0}+\Lambda_{k}^{s}\left(t-(k-1) T_{s}\right), \\
& \left.\quad k \in \mathbb{N}: k \leq \bar{k}, \quad t \in](k-1) T_{s}, k T_{s}\right] \\
L(t)= & \left.\left.\Lambda_{k}^{0}+\Lambda_{k}^{s}\left(t-\bar{k} T_{s}\right), \quad t \in\right] \bar{k} T_{s}, T\right]
\end{aligned}
$$

where $\bar{k}=\max \left\{k \in N: k<T / T_{s}\right\}$.

Exploiting the Matlab LMI toolbox [9], it is possible to find matrices $\Pi_{k}^{s}, \Pi_{k}^{0}, \Lambda_{k}^{s}$ and $\Lambda_{k}^{0}, k=1,2, \ldots, r$ such that $P(\cdot)$ and $L(\cdot)$ verify the conditions of Theorem 3 . Therefore we can conclude that the closed loop system obtained by the interconnection of (19) and (20), with $K(t)=L(t) Q^{-1}(t)$ is FTS with respect to $(T, R, \Gamma)$. The time behavior of the two scalar functions $K_{1}(t)$ and $K_{2}(t)$, $K(t)=\left(K_{1}(t) \quad K_{2}(t)\right)$, is shown in Fig. 3 .

\section{CONClusions}

This paper has dealt with the finite-time stability problem for linear time-varying systems with jumps. A necessary and sufficient condition is found by exploiting the transition matrix properties; however such condition is numerically hard to solve unless we deal with time-invariant systems; moreover it is not useful for dealing with the synthesis problem. Therefore a sufficient condition, involving two coupled D/DLMIs, has been given. The effectiveness of the proposed condition has been evaluated on a closed loop sampled-data system, suggesting that the degree of conservativeness remains acceptable. Finally, the analysis result has been exploited to derive synthesis conditions for the design of a state-feedback controller, again based on coupled D/DLMIs. When the structure of the matrix variable $P(\cdot)$ (or $Q(\cdot)$ ) is a priori fixed, both the analysis 
and synthesis problems can be converted into a classical LMIs optimization problem, allowing the resolution by means of efficient off-the-shelf optimization algorithms.

\section{REFERENCES}

[1] F. Amato, M. Ariola, M. Carbone, and C. Cosentino. Finite-time output feedback control of linear systems via differential linear matrix conditions. In Proc. Conference on Decision and Control, pages 5371-5375, San Diego, CA, 2006.

[2] F. Amato, M. Ariola, and C. Cosentino. Finite-time control of linear time-varing systems via output feedback. In Proc. American Control Conference, pages 4722-4726, Portland, OR, 2005.

[3] F. Amato, M. Ariola, and C. Cosentino. Finite-time stabilization via dynamic output feedback. Automatica, 42:337-342, 2006.

[4] F. Amato, M. Ariola, and P. Dorato. Finite time control of linear systems subject to parametric uncertanties and disturbances. Automatica, 37:1459-1463, 2001.

[5] P. J. Antsaklis. Hybrid Systems II. Springer, 1995.

[6] R. Boel and G. Stremersch. Discrete Event Systems: Analysis and Control. Springer, 2000.

[7] S. Boyd, L. El Ghaoui, E. Feron, and V. Balakrishnan. Linear Matrix Inequalities in System and Control Theory. SIAM Press, 1994.

[8] P. Dorato. Short time stability in linear time-varying systems. In Proc. IRE International Convention Record Part 4, pages 83-87, 1961.

[9] P. Gahinet, A. Nemirovski, A. J. Laub, and M. Chilali. LMI Control Toolbox. The Mathworks Inc, 1995.

[10] W. Sun, K. Nagpal, and P.P. Khargonekar. $\mathcal{H}_{\infty}$ control and filtering for sampled-data systems. Ann Arbor, 1993.

[11] L. Weiss and E. F. Infante. Finite time stability under perturbing forces and on product spaces. IEEE Trans. Auto. Contr., 12:54-59, 1967.

[12] T. Yang. Impulsive Control Theory. Springer, 2001. 